\title{
La gestión municipal de los castillos valencianos. Análisis de los casos de Callosa de Segura, Castalla, Cocentaina, Guardamar del Segura, Monòver, Pego y Sax (provincia de Alicante) Juan Antonio Mira Rico
}

Servei Municipal de Patrimoni Cultural de Castalla, Castalla, España, mirarico@ hotmail.com

\begin{abstract}
Valencian castles have been the subject of research and have undergone multiple architectural interventions, which have allowed getting an understanding of them, improving their condition, and restoring them. However, it should be noted a lack of research addressing their management. A PhD thesis project has been carried out since 2013 aiming at comprehending how the city councils of Alicante province run their fortifications. The methodology used consists of qualitative research and semi-structured interviews with experts (municipal technicians). Since the project is still ongoing, for the time being only preliminary results of the analysis of one of the 8 groups into which castles have been divided, specifically those belonging to group 4, will be published. This group of fortifications, located in cities with populations that range between 10,000 and 20,000, is comprised of Ambra (Pego), Callosa de Segura, Castalla, Cocentaina, Guardamar del Segura, Monòver, Penella (Cocentaina) and Sax castles.
\end{abstract}

Keywords: castles, cultural heritage management, towns, Alicante province.

\section{Introducción}

Los castillos valencianos, en su mayoría de propiedad pública y, sobre todo, municipal, han sido, en mayor o menor medida, objeto de estudio desde muy diversos enfoques por el gran interés que despiertan entre la sociedad. De esta manera, se ha podido conocer mejor su historia, su morfología, los materiales empleados en su construcción, las patologías que sufren; así como aspectos relativos a las gentes que los ocuparon en distintos momentos de la historia.

Igualmente, se han ejecutado múltiples actuaciones, de conservación y/o restauración; que han servido para mejorar su estado y recuperarlos para usos acordes con los tiempos actuales. En especial destacan las actuaciones de didáctica y difusión, realizadas para lograr su conversión en productos turístico-culturales. Existen muchos ejemplos de ello en la provincia de Alicante.

Es el caso, por ejemplo, de fortificaciones ${ }^{1}$ incluidas en este trabajo como el Castell de Castalla (Menéndez et. al.: 2010), restaurado entre los años 2003 y 2006; y de otras que forman parte de la tesis doctoral que da pie a este estudio como el Castell de Biar (Azuar, 1981 e Hinojosa, 1995).

Este último castillo ha sido objeto de diversas intervenciones de consolidación y restauración entre la $2^{\mathrm{a}} 1 / 2$ del siglo XX y la primera década del siglo XXI (Azuar, 1981; del Rey y Gallud, 2010: 429; y Segura y Simón, 2001: 55 y 56). 
2. ¿Por qué una investigación para conocer cómo gestionan los Ayuntamientos de la provincia de Alicante los castillos de su propiedad?

Porque, a pesar del interés que despiertan los castillos, de las intervenciones realizadas en ellos y de los múltiples trabajos publicados; todavía no se ha analizado en el contexto de la Comunidad Valenciana, en general, y en el de la provincia de Alicante, en particular, cómo administran los ayuntamientos alicantinos las fortificaciones de su propiedad. En este sentido, desde el año 2013, se desarrolla una tesis doctoral, en el seno del Área de Prehistoria de la Universidad de Alicante y en curso en el momento de redactar este trabajo, con la cual se pretende rellenar el vacío que actualmente existe en este campo. En la misma se analiza la gestión que los municipios de la provincia de Alicante han realizado de los castillos de su propiedad entre los años 2003 y 2013.

Teniendo presente lo anterior, se han formulado los siguientes objetivos:

- General. Conocer cómo han administrado los municipios de la provincia de Alicante las fortificaciones de su propiedad desde 2003 hasta 2013.

- Específicos. Saber qué personas (técnicos en materia de patrimonio cultural, turismo, directores de museos, etc.) y qué áreas y/o instituciones municipales (servicios de patrimonio cultural, servicios de cultura, museos, oficinas de turismo, etc.); se han encargado de gestionar los castillos analizados; identificar las semejanzas y diferencias, a nivel de administración, que hay entre las distintas fortificaciones estudiadas; y conocer qué actuaciones de investigación, conservación, restauración, didáctica y difusión, se han realizado en los castillos investigados.

A partir de los objetivos formulados se han planteado cuatro preguntas de investigación (Flick, 2004: 61-67) ¿cómo han gestionado los municipios de la provincia de Alicante los castillos de su propiedad?; ¿qué personal técnico los han administrado?; ¿en qué áreas y/o instituciones municipales se ha realizado dicha gestión? y ¿hay un modelo único de administración o, en realidad, hay varios?; que se han plasmado en la siguiente hipótesis de trabajo: el tamaño de los municipios, la formación de los gestores y las áreas y/o instituciones municipales en las cuales se ha desarrollado dicha gestión; han condicionado la administración realizada en los castillos de propiedad municipal situados en la provincia de Alicante, en el periodo comprendido entre 2003 y 2013. La elección de dicho marco temporal obedece a una cuestión de operatividad. Pues, para llevar a buen puerto una investigación con una muestra tan amplia (y, en algunos casos, gestionada durante largo tiempo); es necesario contar unos límites cronológicos precisos, caso de los años escogidos. Además, éstos presentan la ventaja de poder comparar la gestión en el periodo previo a la crisis económica (20032007) y durante la misma (2008-2013).

\section{Metodología}

Para poder cumplir con los objetivos señalados y validar, o refutar, la hipótesis formulada, se ha utilizado la metodología de investigación cualitativa (Flick, 2004; Taylor y Bogdan, 2002; y Vasilachis, 2006). En concreto, se ha empleado la entrevista semiestructurada a expertos municipales. Se ha optado por este modelo porque permite centrar la entrevista, no en la persona, sino en su cualidad de especialista y recoger información sobre el objeto de estudio en cuestión. De esta manera, "el experto se integra en el estudio, no como un caso individual, sino como representación de un grupo (de expertos) (...)"; hecho que permite analizar y comparar "(...) el contenido del conocimiento del experto" (Flick, 2004: $104 \mathrm{y}$ 105). Y es precisamente ese conocimiento el cual interesa para este estudio, porque las personas entrevistadas son las responsables de la gestión de los castillos analizados. No obstante, en el caso de Sax se ha hecho una excepción y también se ha recogido la opinión de un experto que, si bien no trabaja en el Ayuntamiento de Sax y no administra directamente la fortificación, si redactó el plan director de dicho castillo. En cuanto al Castell de Castalla, hay que señalar que la persona entrevistada es la 
misma que ha llevado a cabo este trabajo, pues sólo hay un técnico encargado de la gestión del castillo. Es decir tiene la doble condición de investigador y experto. Desde un punto de vista metodológico ser objeto y sujeto al mismo tiempo de un estudio es extraño. Pero esta situación se ha producido porque no hay ningún técnico más, en el Servei Municipal de Patrimoni Cultural de Castalla, que pueda contestar las preguntas de la entrevista. También se podía haber dejado fuera de la investigación el Castell de Castalla, pero no se ha considerado correcto por su importancia histórica y el trabajo que se está haciendo en él. Dicho esto sólo resta añadir que las preguntas se han contestado sinceramente y que todas las respuestas pueden contrastarse de manera fehaciente con trabajos y datos disponibles en documentos, páginas de Internet y prensa.

No obstante, al igual que las entrevistas estructuradas y las entrevistas en profundidad; la entrevista semiestructurada también presenta ventajas e inconvenientes perfectamente sintetizados por Eloísa Pérez Santos (2000: 96) y Michael Meuser y U. Nagel (en Flick, 2004: 104 y 105). Durante la realización de las entrevistas, ejecutadas entre junio de 2013 y diciembre de 2014, se ha intentado, por todos los medios posibles, evitar dichos hándicaps. En cuanto a sus características propiamente dichas, la entrevista diseñada cuenta con 59 preguntas agrupadas en 5 bloques: datos de la/s persona/as entrevistada/as; datos de gestión generales; datos de gestión: investigación; datos de gestión: conservación; datos de gestión: restauración; y datos de gestión: didáctica y/o difusión.

\subsection{Criterios de selección de la muestra analizada}

Los principios establecidos, estimados como los más idóneos, para obtener la muestra necesaria para llevar a cabo el estudio propuesto, han sido: el análisis de las fortificaciones, consideradas como tales según el Inventario General del Patrimonio Cultural Valenciano (por ello no se han estudiado construcciones que, aunque tengan el nombre de castillos, en realidad corresponden a otro tipo de arquitectura); el estudio de las fortificaciones no enmascaradas por obras posteriores, como el Castell de Benidorm (Benidorm), localizado bajo la actual Plaza del Castillo; el establecimiento de un marco geográfico de análisis concreto: la provincia de Alicante (un lugar privilegiado para el estudio de los castillos en cualquiera de sus vertientes y que cuenta con 57 fortificaciones públicas y privadas); el análisis de los castillos de titularidad y gestión pública, ya que el objetivo general de este trabajo es conocer cómo administran los municipios de la provincia de Alicante las fortificaciones de su propiedad. Por ello, no se han estudiado los castillos de propiedad privada.

Los 48 castillos analizados en la tesis doctoral, se han agrupado en entornos similares y no distintos. Porque no tiene sentido comparar la gestión que realiza de su fortificación Castalla, un municipio de tamaño medio; con la que lleva a cabo de la suya Alicante, capital de la provincia. Los recursos, en todos los niveles, no pueden ser, lógicamente, los mismos. El criterio para establecer los entornos similares ha sido el poblacional, pues municipios de semejante tamaño deberían disponer, al menos en principio y en teoría, de unos recursos y capacidades similares para gestionar sus fortificaciones (aunque, a la hora de la verdad, la realidad suele variar de la teoría). Así pues, aprovechando la distribución realizada por el INE, que clasifica el tamaño de los ayuntamientos en función de sus habitantes, los municipios alicantinos se han dividido en 8 grupos. Y los castillos objeto de estudio se han clasificado en cada uno de los 8 grupos. Las 8 fortificaciones incluidas en el presente trabajo, se incluyen en el grupo 4, formado por los municipios de Callosa de Segura, Castalla, Cocentaina, Guardamar del Segura, Monòver, Pego y Sax, que cuentan entre 10.001 y 20.000 habitantes.

\subsection{Criterios de selección de las personas entrevistadas}

Tras seleccionar y distribuir los castillos de interés en sus correspondientes grupos, el siguiente paso ha sido determinar los expertos que debían ser entrevistados. Los requisitos para 
ello era contar con un perfil técnico y desarrollar su labor en los ayuntamientos responsables de las diferentes fortificaciones (independientemente de si son o no funcionarios). En este sentido, la figura más idónea para ser entrevistada es la de técnico en patrimonio cultural. No obstante, al tratarse de un perfil laboral joven, la mayor parte de los ayuntamientos no cuentan con la misma en sus plantillas. Por ello, en la mayoría de los casos, se ha tenido que recurrir a otros expertos. Centrando la atención en el grupo 4, las personas seleccionadas para ser entrevistadas han sido las siguientes: Castell d'Ambra (archivero y arquitecto municipal de Pego); Castillo de Callosa de Segura (director de los Museos Municipales de Callosa de Segura); Castell de Castalla (responsable técnico del Servei Municipal de Patrimoni Cultural de Castalla); castells de Cocentaina y Penella (técnico en patrimonio cultural de Cocentaina); Castillo de Guardamar del Segura (conservador del patrimonio cultural de Guardamar del Segura); Castell de Monòver (responsable técnico del Servicio de Obras, Urbanismo y Medio Ambiente); y Castillo de Sax (catedrático de Geografía Humana en la Universidad de Alicante, auxiliar de Cultura, archivero y bibliotecario de Sax, arquitecto municipal y técnico en medio ambiente de Sax). Se han dejado de lado otros perfiles, como el político, porque al no tratarse de expertos, no se han considerado óptimos para dicho trabajo.

Como puede apreciarse se ha identificado una variada gama de técnicos que participan en la administración de las fortificaciones estudiadas.

\section{Características de la muestra analizada}

Como ya se ha expuesto, los castillos estudiados en esta publicación forman del grupo 4 (de 8 existentes) en la investigación en curso para conocer cómo han gestionado los Ayuntamientos de la provincia de Alicante las fortificaciones de su propiedad en el periodo comprendido entre 2003 y 2013.

El trabajo completo contiene una ficha con diversos datos de interés sobre los castillos: adscripción cronológica y cultural, si son o no visitables, entre otros aspectos. Por cuestiones obvias las fichas no se incluyen aquí. No obstante, en este apartado se han incluido diversas referencias bibliográficas para conocer mejor cada fortificación: Castell d'Ambra (Azuar y Navarro, 1995); Castillo de Callosa de Segura (Azuar, 1981; e Hinojosa, 2001); Castell de Castalla (Hinojosa, 2001; y Menéndez et al., 2010); Castell de Cocentaina (Domenech, 2003; Ferrer y Català, 1996; e Hinojosa, 2001); Castillo de Guardamar del Segura (AA.VV., 2010; Azuar, 1981 e Hinojosa, 1996); Castell de Monòver (Ortega y Esquembre, 2001): Castell de Penella (Domenech, 2003; Ferrer, 1996; e Hinojosa, 2001); y Castillo de Sax (Azuar, 1981; Hinojosa, 2001; y Simón y Segura, 2001 b).

\section{Análisis de las entrevistas a expertos}

En este punto se han analizado las respuestas, en cada uno de los 6 bloques mencionados el punto 3 , con la intención de obtener una visión de conjunto de cada una de las cuestiones planteadas, poder cumplir con los objetivos señalados y verificar o descartar la hipótesis formulada. Por cuestiones de espacio, en cada uno de los bloques sólo se recogen las cuestiones que se han juzgado más interesantes.

\subsection{Bloque 1. Datos de las personas entrevistadas}

- De las 13 entrevistas realizadas, 12 personas gestionan fortificaciones, mientras que 1 (archivero municipal de Pego), no participa en la gestión de Ambra. De las 12 personas que administran castillos, 5 desempeñan labores de técnicos en patrimonio cultural y/o similares (Callosa de Segura, Castalla, Cocentaina, Guardamar del Segura y Penella); 3 ejercen de arquitectos (Ambra, Monòver y Sax); 1 es auxiliar de cultura (Sax); 1 es archivero y bibliotecario (Sax); 1 es técnico en medio ambiente; y 1 es profesor universitario (Sax). Sólo una de las personas entrevistadas no participa en la gestión de la fortificación de su municipio (Pego).

- Únicamente en los casos de Castalla, Cocentaina, Penella y Sax (auxiliar de cultura y profesor universitario por partida doble); las personas entrevistadas, tienen formación 
específica en administración del patrimonio cultural.

\subsection{Bloque 2. Datos de gestión generales}

- De las 8 fortificaciones analizadas, 5 (Ambra, Callosa de Segura, Cocentaina, Penella y Sax) dependen de las concejalías de cultura de sus respectivos ayuntamientos. Castalla y Guardamar del Segura dependen de las concejalías de patrimonio cultural (Concejalía de Patrimonio Cultural y Concejalía de Patrimonio Arqueológico, respectivamente). No se ha podido determinar la concejalía titular de Monòver por desconocimiento de la persona entrevistada (responsable técnico del Servicio de Obras, Urbanismo y Medio Ambiente).

- Sólo Castalla y Sax cuentan con herramientas de administración. En el caso de Castalla, un plan de gestión que se inició en el año 2009 y todavía se encuentra en vigor (aunque la idea es sustituirlo por un plan director). Sax cuenta con un plan director que no se encuentra en vigor por cuestiones políticas (según información facilitada por el catedrático de Geografía en la Universidad de Alicante). Ambra, Cocentaina, Guardamar del Segura, Monòver y Penella carecen de ellos por falta de iniciativa y/o voluntad política. En el caso de Callosa de Segura no se ha realizado ninguno "porque no se ha planteado", en la palabras de la persona entrevistada (director de los Museos Municipales de Callosa de Segura).

- La utilidad de las herramientas de gestión ha sido valorada positivamente, salvo en Ambra (archivero municipal); y Monòver (responsable técnico del Servicio de Obras, Urbanismo y Medio Ambiente).

- Solamente Castalla cuenta con un equipo interdisciplinar que participa en su gestión ${ }^{2}$.

- Únicamente Castalla y Sax cuentan con subvenciones específicas de organismos públicos (Ayuntamientos).

- Los puntos fuertes y débiles de todos los castillos son comunes y se encuentran en valores como la secuencia histórica y la accesibilidad.
- Sólo Castalla y Sax cuentan con estudios evaluativos. Para el año 2013 presentaban cifras similares: 2.058 visitantes en Castalla y 2.239 en Sax.

\subsection{Bloque 3. Datos de gestión: investigación}

- De las fortificaciones estudiadas, en 4 de ellas (Castalla, Cocentaina, Penella y Sax); se han realizado actuaciones de investigación, con predominio de las de tipo histórico (actuaciones arqueológicas, análisis de documentación, etc.)

- En todas ellas los resultados de las actuaciones de investigación han sido los esperados, porque las intervenciones han permitido, entre otros aspectos, mejorar el conocimiento que se tenía de ellos. Pero, solamente en Castalla está previsto continuar investigando.

\subsection{Bloque 4. Datos de gestión: conservación}

- Salvo en Ambra y Callosa de Segura, en el resto de castillos se han realizado actuaciones de conservación.

- En todos ellos se han conseguido los resultados esperados porque dichas intervenciones han contribuido a mantener en buen estado las fortificaciones (o evitar que su deterioro fuera a más). Sólo en tres casos, Callosa de Segura, Castalla y Guardamar del Segura, se piensa continuar con dichos trabajos. En el resto no, por diversas razones: falta de gestión e implicación política (Ambra y Monòver), porque los problemas se han solucionado (Cocentaina y Penella); y porque todo se encuentra en perfecto estado (Sax).

\subsection{Bloque 5. Datos de gestión: restauración}

- Solamente en Castalla y Penella se han ejecutado actuaciones de restauración.

- En Castalla se procedió a su restauración completa, mientras que en Penella se llevó a cabo una restauración parcial, así como una mejora del acceso.

- Tanto en Castalla como en Penella se han producido los resultados esperados. En Castalla, porque se ha frenado su deterioro, se ha mejorado su estado de conservación y se ha 
facilitado su conversión en producto turísticocultural. Y en Penella, porque se ha evitado su desaparición.

- En Castalla y Penella no se llevarán a cabo actuaciones de este tipo porque han sido restaurados recientemente, entre los años 20032006 y 2006, respectivamente. En Cocentaina tampoco, porque la parte principal del castillo se encuentra restaurada. En Sax no "por falta de recursos económicos" (según el arquitecto municipal) y porque se encuentra bastante restaurado, hecho que no implica que haya que restaurar más. Finalmente, en Ambra, Callosa de Segura, Guardamar del Segura y Monòver no está previsto realizar ninguna intervención de este tipo.

\subsection{Bloque 6. Datos de gestión: didáctica y/o difusión}

- Salvo en Monòver, en el resto de fortificaciones gestionadas se han ejecutado intervenciones de este tipo que, por otro lado, son las más numerosas.

- No obstante, hay castillos con una enorme actividad, caso de Castalla y Sax. Otros, como Callosa de Segura, Cocentaina, Guardamar del Segura y Penella, presentan, en cambio, un menor movimiento. Además, Castalla y Sax son las únicas fortificaciones de este grupo que forman parte de la Ruta de los Castillos del Vinalopó (http://www.rutacastillosvinalopo.net).

- En todos los castillos en los cuales se han llevado actuaciones se han producido los resultados esperados. Todas las respuestas coinciden, en líneas generales, en que este tipo de intervenciones ayudan a conocer y difundir mejor el castillo.

- Salvo en Callosa de Segura, Guardamar del Segura y Monòver, en el resto de fortificaciones se apuesta por la realización de nuevas actuaciones (visitas punturales, visitas guiadas, publicaciones científicas y divulgativas, etc.) de estas características.

- De todos los castillos, sólo Cocentaina, Guadamar del Segura, Penella y Sax están musealizados. En Cocentaina y Penella la musealización es completa, en tanto que en Guardamar del Segura y Sax es parcial.
Cocentaina, Penella y Sax, poseen una musealización tradicional, mientras que la de Guardamar del Segura es interactiva ${ }^{3}$. El resto de fortificaciones (Ambra, Callosa de Segura, Castalla y Monòver), no están musealizadas.

- En los castillos musealizados, los técnicos entrevistados optarían por los siguientes tipos de musealización: mixta (interactiva y tradicional), en Cocentaina y Penella, e interactiva y didáctica, en Guardamar del Segura y Sax.

\section{Conclusiones}

A través de este trabajo y de la tesis doctoral de la cual forma parte, inédito desde el punto de vista académico, se ha podido conocer $\mathrm{y}$ profundizar en la administración que los municipios de entre 10.001 y 20.000 habitantes de la provincia de Alicante han realizado en los castillos de su propiedad entre los años 2003 y 2013. No obstante, al tratarse de una investigación todavía en curso, este punto debe tomarse como provisional hasta la finalización de la misma. Dicho esto destacan las siguientes conclusiones:

- Se ha alcanzado el objetivo general del trabajo. Así los municipios de Castalla (Castell de Castalla), Cocentaina (Castell de Cocentaina y Castell de Penella) y Sax (Castillo de Sax); han administrado sus fortificaciones. En cambio, los municipios de Callosa de Segura (Castillo de Callosa de Segura), Guardamar del Segura (Castillo de Guardamar del Segura), Monòver (Castell de Monòver) y Pego (Castell d'Ambra) no los han gestionado (o la administración ha sido mínima), entre los años 2003 y 2013, marco temporal de este estudio. Por lo tanto, puede que anteriormente los castillos se administrasen, o que se gestionen en el futuro. No obstante, que haya castillos no administrados, o poco gestionados, no deja de ser, cuanto menos, sorprendente. Cualquier actuación, por pequeña y modesta que sea, siempre es mejor que dejar en el abandono nuestras fortificaciones.

- Igualmente se han conseguido los objetivos específicos establecidos. Al determinar qué personas (técnicos en materia de patrimonio cultural, turismo, directores de museos, etc.,) y qué áreas y/o instituciones municipales 
(servicios de patrimonio cultural, servicios de cultura, museos, oficinas de turismo, etc.); se han encargado de gestionar los castillos analizados; identificar las semejanzas y diferencias, a nivel de administración, que ha habido entre las distintas fortificaciones estudiadas; y conocer qué actuaciones de investigación, conservación, restauración, didáctica y difusión, se han realizado en las castillos investigados. En esta línea puede afirmarse que, en los castillos administrados, no existe un único modelo de gestión. Más bien al contrario, cada fortificación sigue un camino independiente. Por ejemplo, sólo dos castillos (Castalla y Sax) cuentan con herramientas de administración. Este hecho debe servir para reflexionar, porque si se tiene en cuenta la relación histórica que muchos castillos guardan entre sí, puede plantearse el establecimiento de un modelo de gestión conjunto en, al menos, determinados aspectos, como la didáctica y la difusión. En este sentido, por ejemplo, las fortificaciones de Castalla y Sax, integrantes de la Ruta de los Castillos del Vinalopó, mantienen una relación estrecha porque ambos castillos formaron parte de la frontera de las Coronas de Aragón y Castilla durante la Edad Media, respectivamente. De esta manera pueden elaborarse contenidos comunes para visitas guiadas y/o autoguiadas o musealizaciones complementarias entre sí. Aquí conviene señalar que, en la provincia de Alicante, hay pocos castillos musealizados. Es necesario musealizar más fortificaciones apostando por nuevos modelos como el interactivo.

- Se ha validado la hipótesis planteada en el punto 2. Como se ha demostrado a lo largo de las páginas precedentes, el tamaño de los municipios, la formación de los gestores y las áreas y/o instituciones municipales en las cuales se desarrolla dicha administración; condicionan la gestión que se ha realizado en los castillos de propiedad municipal, situados en la provincia de Alicante, entre 2003 y 2013. Tomando como ejemplo el Castell d'Ambra y el Castell de Castalla, ambas fortificaciones se encuentran en municipios con una población similar (Pego contaba, en el año 2013, con 10.957 habitantes empadronados y Castalla tenía 10.579); la persona que gestionaba el Castell d'Ambra es arquitecto, sin formación específica en administración del patrimonio cultural, mientras que el gestor del Castell de Castalla es historiador con formación concreta en gestión del patrimonio cultural; finalmente el Castell de d'Ambra depende de la Concejalía de Cultura, y el Castell de Castalla está bajo control la Concejalía de Patrimonio Cultural. En el caso del Castell de Castalla, la suma de todos estos factores, más el apoyo activo del Ayuntamiento de Castalla, ha producido resultados muy positivos en la administración de la fortificación.

- Tanto este trabajo, como su versión más extensa, complementan la investigación histórica de la cual han sido objeto, tradicionalmente, los castillos. Sus resultados son el punto de partida para formular nuevos planteamientos de administración que permitan mejorar en unos casos, iniciar en otros, la gestión de las fortificaciones analizadas. Además, no se trata de un tema agotado, al contrario. En el futuro se podrá extender a otros territorios.

\section{Notas}

(1) Se utiliza indistintamente, como sinónimos, los términos castillo y fortificación; así como gestión y administración.

(2) Más información en http://www.gestioncultural.org/buenas_practicas .php?id_proyectos=299223.

(3) La musealización tradicional se basa en la primacía del objeto que expone, en el papel pasivo del visitante y en su enfoque, en muchas ocasiones, para especialistas. Por el contrario, la musealización interactiva favorece la participación activa del visitante, es accesible para todos, mucho más didáctica (aunque no por ello menos rigurosa), y emplea más recursos que los objetos expuestos para dar a conocer los contenidos. Ambos modelos presentan ventajas y desventajas, pero puestos a musealizar, lo mejor es apostar por una musealización para todos (y no solamente para los especialistas); que favorezca el disfrute y el aprendizaje de la gente fuera de las aulas. Y en este sentido, la museografía interactiva va por delante de la tradicional. 


\section{Referencias}

AA.VV. (2010): Guardamar del Segura. Arqueología y Museo. MARQ Ed. Alicante. p. 295.

Azuar, R. (1981). Castellología medieval alicantina: área meridional. Diputación de Alicante Ed. Alicante. p. 250.

Azuar Ruiz, R., Navarro Suárez, F. J. 1995. Castillos de Alicante. Lancia Ed. León. p. 92.

Ferrer, P., Català, F. 1996. El Comtat: una terra de castells. Centre d'Estudis Contestans Ed. Cocentaina. p. 122.

Doménech, E. coord. (2003). El patrimoni històric i artístic de Cocentaina. La seua recuperació. Les intervencions Arquitectòniques i Arqueològiques. Ajuntament de Concentaina Ed. Cocentaina. p. 501.

Flick, U. (2012). Introducción a la investigación cualitativa. Morata Ed. Madrid. p. 324.

Hinojosa, J. R. (1996). "Guardamar: un castillo en ruinas a finales de la Edad Media" in Castells. Asociación Española de Amigos de los Castillos. Sección provincial de Alicante Ed. Alicante. pp. 19-22.

Hinojosa, J. R. (2001). Diccionario de historia medieval del reino de Valencia. Generalitat Valenciana Ed. Valencia. p. 2675.

Menéndez, J. L., Bevià, M., Mira, J. A., Ortega, J. R. (2010). El Castell de Castalla. Arqueología, arquitectura e historia de una fortificación de frontera. MARQ Ed. Alicante. p. 309.

Ortega, J. R., Esquembre, M. A. (2001). "Castillo de Monóvar (Medio Vinalopó)" in Castillos y torres en el Vinalopó. Centre d'Estudis Locals del Vinalopó. Ed. Petrer. pp. 133-137.

Pérez, E. (2000). Estudio de visitantes en museos: metodología y aplicaciones. TREA Ed. Gijón. p. 256.

Del Rey, M., Gallud, A. (2010). "Intervención y consolidación del segundo recinto amurallado del Castillo de Biar" in Arché. Universitat Politécnica de València Ed. València. pp. 427-438.

Segura, G., Simón, J. L. (2001): "Castillo de Biar (Alto Vinalopó)" in Castillos y torres en el Vinalopó. Centre d'Estudis Locals del Vinalopó. Ed. Petrer. pp. 55-59.

Simón, J. L., Segura, G. (2001). "El Castillo de Sax (Alto Vinalopó)" in Castillos y torres en el Vinalopó. Centre d'Estudis Locals del Vinalopó. Ed. Petrer. pp. 89-99.

Taylor S. J., Bogdan, R. (2002): Introducción a los métodos cualitativos de investigación. Paidos Ibérica. Ed. Barcelona. p. 344.

Vasilachis, I. coord. (2009). Estrategias de investigación cualitativa. Gedisa Ed. Barcelona. p. 28 